Proyecciones Journal of Mathematics

Vol. 38, No 2, pp. 237-253, June 2019.

Universidad Católica del Norte

Antofagasta - Chile

\title{
On Fuzzy $\Lambda_{\gamma}$-Sets and their Applications
}

\author{
Baby Bhattacharya \\ NIT Agartala, India \\ Arnab Paul \\ Techno College of Engineering Agartala, India \\ and \\ Jayasree Chakraborty \\ NIT Agartala, India \\ Received: June 2017. Accepted: March 2019
}

\begin{abstract}
The notion of $\Lambda$-fuzzy set was introduced by M. E. EI-Shafei and A. Zakari in 2006 [ $\theta$-Generalized closed sets in fuzzy topological spaces, The Arabian Journal for Science and Engineering, Vol. 31, Number 2A]. We examine some basic properties of it and prove some characterization theorems for the same. The paper presents a new class of fuzzy sets called fuzzy $\Lambda_{\gamma}$-sets that includes the class of all fuzzy $\gamma$-open sets. It also introduces the notion of fuzzy $V_{\gamma}$-sets as the dual concept of fuzzy $\Lambda_{\gamma}$ sets to study the spaces constituted by those sets and obtain a completely different structure which is called fuzzy independent Alexandorff space. A stronger form of fuzzy $\Lambda_{b}$ continuity [Gulhan Aslim, Gizem Gunel On fuzzy $\Lambda_{b}$-sets and fuzzy $\Lambda_{b}$-continuity. Chaos, Solitons and Fractals 42 (2009). 1024-1030] called fuzzy $\Lambda_{\gamma}$-continuity is introduced and the relationships are also established with the already existing functions accordingly. Finally, fuzzy $\Lambda_{\gamma}$-Generalized closed sets are defined and studied with some of their applications.
\end{abstract}

Keyword: Fuzzy $\gamma$-open set, Fuzzy independent topology, Fuzzy $\Lambda_{b}$ set, Fuzzy $\Lambda_{\gamma}$-set, Fuzzy $\Lambda_{b}$-continuity, Fuzzy $\Lambda_{\gamma}$-closed set. 


\section{Introduction}

The potential of the notion of fuzzy set studied by L. A. Zadeh [24] was realized by the researchers and has successfully been applied for new investigations in all the branches of science and technology for more than last five decades. Since Chang [7] defined the concept of a fuzzy topology, then many authors investigated different properties of fuzzy open sets which are weaker than the property of openness of a fuzzy set in a fuzzy topological space. For example, ([19],[3],[25],[21]) have considered such kind of properties of fuzzy sets and most of the collection forms a fuzzy supra topology therein. A significant contribution to the theory of generalized open sets has been reported by A. Csaszar ([8], [9], [10]) and extended by G. Palani Chettry [16] in the context of fuzzy set theory with the name of generalized fuzzy topological space. For example, $([19],[3],[25],[21])$ have considered such kind of properties of fuzzy sets and most of the collection forms a fuzzy supra topology therein. A significant contribution to the theory of generalized open sets has been reported by A. Csaszar ([8], [9], [10]) and extended by G. Palani Chettry [16] in the context of fuzzy set theory with the name of generalized fuzzy topological space. Very recently, a different but unique form of fuzzy open sets is being proposed by B. Bhattacharya [6] and established by defining the concept of fuzzy $\gamma$-open sets in the sense of D. Andrijevic[1], which are called fuzzy independent open sets as they are totally incomparable with the fuzzy open sets. Also, it is shown that the collection of all fuzzy $\gamma$-open sets forms a fuzzy topology itself but does not contain the class of fuzzy open set or not contained in the given fuzzy topology. A finer topology namely Alexandorff topology is established in graphs studied by Jafarian et al. [12] in 2013. It becomes a common approach to study the generalization of topological concepts for a quite long time, for instance $([11],[13])$. Our aim is to study the parallel concept of topology in a given fuzzy space with an incomparable nature. More recent works by A. Paul et al. are there in the literature to show different applications of fuzzy $\gamma$-open sets ([17], [18]) in the direction of fuzzy bitopology.

In the present paper, we introduce the concept of fuzzy $\Lambda_{\gamma}$-set (resp. fuzzy $\mathrm{V}_{\gamma}$-set) and study some of their properties. We offer a finer fuzzy topology on $\mathrm{X}$ than $\tau_{\gamma}$-by utilizing the new notion of fuzzy $\Lambda_{\gamma}$-sets. Finally, we discuss about some fundamental properties of such structure and some related maps. In particular, we have shown that fuzzy $\Lambda_{\gamma}$-continuity is a stronger form of fuzzy $\Lambda_{b}$-continuity introduce by Aslim et al.[2] in 2009. Lastly, we define fuzzy $\Lambda_{\gamma}$-generalized closed set and related functions with 
some comparisons related functions with some comparisons.

As El Nachic [14] contemplated that the concept of fuzzy topology may be relevant to quantum physics in connection with string theory and $\epsilon^{\infty}$ theory, we claim that the results given in the present paper may turn out to be useful in quantum physics.

Let $\mathrm{X}$ be any set and $\mathrm{I}$ be the closed unit interval $[0,1]$. A fuzzy set of $\mathrm{X}$ is an element of the set of all functions from $\mathrm{X}$ into I. The family of all fuzzy sets in $\mathrm{X}$ is denoted by $\mathrm{I}^{X}$.A fuzzy point $\mathrm{x}_{p}[23]$ is a fuzzy set in $\mathrm{X}$ defined by $\mathrm{x}_{p}(\mathrm{x})=\mathrm{p}, \mathrm{x}_{p}(\mathrm{y})=0$ for al $x \neq y, p \in[0,1]$ and $x_{p} \in \mu$ iff $p \leq \mu(x)$.

Throughout this work, by $(\mathrm{X}, \tau)$ we mean a fuzzy topological space (for short, fts) due to Chang [7] in 1968. The complement of a fuzzy set $\mu$ is denoted by $\mu^{C}$. We consider the following results and definitions for ready references.

\subsection{Definition}

Let $\mu$ be a fuzzy subset of a fts $(\mathrm{X}, \tau)$. Then $\mu$ is called a fuzzy pre-open set [19] if $\mu \leq \operatorname{int}(\operatorname{cl}(\mu))$.

\subsection{Definition}

A subset $\lambda$ of a fts $(\mathrm{X}, \tau)$ is said to be fuzzy $\gamma$-open set [6] $\lambda \wedge \mu \in F P O(X)$ for each fuzzy pre-open set $\mu$ in $\mathrm{X}$. The complement of a fuzzy $\gamma$-open is called a fuzzy $\gamma$-closed set.

For any fuzzy set $\delta, \operatorname{int}_{\gamma}(\delta)$ is the union of all $\gamma$-open sets contained in $\delta$ in a fts $(\mathrm{X}, \tau)$. The family of all fuzzy $\gamma$-open sets is denoted by $\mathrm{F} \gamma \mathrm{O}(\mathrm{X})$ and that of fuzzy $\gamma$-closed set is denoted by $\mathrm{F} \gamma \mathrm{C}(\mathrm{X})$.

\subsection{Definition}

A fuzzy subset $\mu$ of a fts $(\mathrm{X}, \tau)$ is called a $\Lambda$-fuzzy set [20] if $\mu=\mu^{\Lambda}$, where $\mu^{\Lambda}=\wedge\{\eta: \mu \leq \eta, \eta \in \tau\}$.

\subsection{Proposition}

Let $(\mathrm{X}, \tau)$ be a $\mathrm{FT}_{1}$ space. Then every fuzzy subset of $\mathrm{X}$ is a $\Lambda$-fuzzy set. 


\subsection{Definition}

A fuzzy set $\eta$ in a fts $(\mathrm{X}, \tau)$ is called fuzzy dense if there exists no fuzzy closed set $\mu$ in $(\mathrm{X}, \tau)$ such that $\mu>\eta$, that is $\operatorname{cl}(\eta)=1_{X}$.

\subsection{Definition}

$[4]$

(i) A fuzzy subset $\lambda$ of a fts $(\mathrm{X}, \tau)$ is called a generalized fuzzy closed (gfc, for short) fuzzy set if $\lambda \leq \eta$ and $\eta \in \tau$ implies that $\operatorname{cl}(\lambda) \leq \eta$.

(ii) $\mathrm{A}$ fts $(\mathrm{X}, \tau)$ is called fuzzy $\mathrm{T}_{1} / 2$ iff every generalized fuzzy closed set in $\mathrm{X}$ is fuzzy closed set in $\mathrm{X}$.

\subsection{Definition}

A fuzzy set $\eta$ in a fts (X, $\tau$ ) is said to be fuzzy locally closed if $\eta=\mu \wedge \beta$, where $\beta$ is a fuzzy closed set and $\mu$ is a fuzzy open set in $\mathrm{X}$.

\subsection{Definition}

$[2]$

Let $f: X \rightarrow Y$ be a function from a fts $(\mathrm{X}, \tau)$ into a fts $(\mathrm{Y}, \sigma)$. Then the function $f$ is called fuzzy $\Lambda_{b}$-continuous if $f^{-1}(\beta)$ is a fuzzy $\Lambda_{b}$-set of $\mathrm{X}$ for each $\beta \in \sigma$.

\section{Spome Properties of $\Lambda$ - Fuzzy Sets}

The notion of $\Lambda$-fuzzy sets [20] were introduced by M.E. El-Shafei and A. Zakari in 2006. This section isdevoted to study some basic properties of $\Lambda$-fuzzy sets to obtain a new kind of fuzzy space. We denote the class of all the $\Lambda$-fuzzy sets by $\mathrm{F} \Lambda(\mathrm{X})$ in $(\mathrm{X}, \tau)$.

\subsection{Lemma}

For fuzzy subsets $\mu, \beta$ and $\left\{\mu_{i}: \mathrm{i} \in \Gamma\right\}$ of a fts $(\mathrm{X}, \tau)$ the following properties hold:

(i) $\Lambda\left(0_{X}\right)=0_{X}$ and $\Lambda\left(1_{X}\right)=1_{X}$.

(ii) $\mu \leq \Lambda(\mu)$.

(iii) $\mu \leq \beta \Rightarrow \Lambda(\mu) \leq \Lambda(\beta)$. 
(iv) $\Lambda(\Lambda(\mu))=\Lambda(\mu)$.

(v) $\Lambda\left(\vee \mu_{i}: \mathrm{i} \in \Gamma\right)=\vee\left\{\Lambda\left(\mu_{i}\right): \mathrm{i} \in \Gamma\right\}$.

(vi) $\Lambda\left(\wedge \mu_{i}: \mathrm{i} \in \Gamma\right) \leq \wedge\left\{\Lambda\left(\mu_{i}\right): \mathrm{i} \in \Gamma\right\}$.

(vii) $\Lambda\left(1_{X}-\mu\right)=1_{X}-\mathrm{V}(\mu)$.

Proof: We only prove (vi) and (vii). The rest parts can be proved in a similar way.

To prove (vi) Let $\mu=\wedge\left\{\mu_{i}: \mathrm{i} \in \Gamma\right\}$. Thus, $\mu \leq \mu_{i}$, for $\mathrm{i} \in \Gamma$.

Hence, from (iii) $\Lambda(\mu) \leq \Lambda\left(\mu_{i}\right)$ for all $\mathrm{i} \in \Gamma$.

$\Rightarrow \wedge\left(\wedge \mu_{i}: \mathrm{i} \in \Gamma\right) \leq \wedge\left\{\wedge\left(\mu_{i}\right): \mathrm{i} \in \Gamma\right\}$.

To prove (vii)

$1_{X}-\mathrm{V}(\mu)=1_{X}-\sup \{\eta: \eta \leq \mu, \eta$ is a fuzzy closed subset of $\mathrm{X}\}$.

$=\inf \left\{\beta: 1_{X}-\mu \leq \beta, \beta\right.$ is a fuzzy open subset of $\left.X\right\}$

$=\wedge\left(1_{X}-\mu\right)$.

\subsection{Theorem}

For fuzzy subsets $\mu$ and $\left\{\mu_{i}: \mathrm{i} \in \Gamma\right\}$ of a fts $(\mathrm{X}, \tau)$ the following properties hold:

(i) $\Lambda(\mu)$ is a $\Lambda$-fuzzy set.

(ii) If $\mu$ is a fuzzy open set, then $\mu$ is $\Lambda$-fuzzy set.

(iii) If $\mu_{i}$ is a $\Lambda$-fuzzy set for each $i \in \Gamma$, then $\vee_{i \in \Gamma} \mu_{i}$ is a $\Lambda$-fuzzy set.

(iv) If $\mu_{i}$ is a $\Lambda$ fuzzy set for each $\mathrm{i} \in \Gamma$, then $\wedge_{i \in \Gamma} \mu_{i}$ is a $\Lambda$-fuzzy set.

Proof: (i) and (ii) are obvious.

(iii) Let $\mu_{i} \in \mathrm{F} \Lambda(\mathrm{X})$ for each $\mathrm{i} \in \Gamma$, then by lemma [2.1(vi)], we get

$\vee_{i \in \Gamma} \mu_{i}=\vee_{i \in \Gamma} \Lambda\left(\mu_{i}\right)=\wedge\left(\vee_{i \in \Gamma} \mu_{i}\right)>\vee_{i \in \Gamma} \mu_{i}$

So, we have $\vee_{i \in \Gamma} \mu_{i}=\Lambda\left(\vee_{i \in \Gamma} \mu_{i}\right)$ and

$\vee_{i \in \Gamma} \mu_{i} \in \mathrm{F} \Lambda(\mathrm{X})$.

(iv) Let $\mu_{i} \in \mathrm{F} \Lambda(\mathrm{X})$ for each $\mathrm{i} \in \Gamma$, then by lemma [2.1(vii)], we get

$\wedge_{i \in \Gamma} \mu_{i}=\wedge_{i \in \Gamma} \Lambda\left(\mu_{i}\right)>\Lambda\left(\wedge_{i \in \Gamma} \mu_{i}\right)>\wedge_{i \in \Gamma} \mu_{i}$.

Thus, we have $\wedge_{i \in \Gamma} \mu_{i}=\Lambda\left(\wedge_{i \in \Gamma} \mu_{i}\right)$ and $\wedge_{i \in \Gamma} \mu_{i} \in \mathrm{F} \Lambda(\mathrm{X})$.

\subsection{Theorem}

The class $\mathrm{F} \Lambda(\mathrm{X})$ of all $\Lambda$-fuzzy sets forms an Alexandroff topology in a fts $(\mathrm{X}, \tau)$ and hence $(\mathrm{X}, \mathrm{F} \Lambda(\mathrm{X}))$ is a fuzzy Alexandroff space.

Proof: (i) $1_{X}, 0_{X} \in \mathrm{F} \Lambda(\mathrm{X})$.

(ii) If $\mu_{i}$ is a $\Lambda$-fuzzy set for each $\mathrm{i} \in \Gamma$, then $\vee_{i \in \Gamma} \mu_{i}$ is a $\Lambda$-fuzzy set, which is proved in the above theorem.

(iii) If $\mu_{i}$ is a $\Lambda$-fuzzy set for each $i \in \Gamma$, then $\wedge_{i \in \Gamma} \mu_{i}$ is a $\Lambda$-fuzzy set,that is proved in the above theorem. 
So the pair $(\mathrm{X}, \mathrm{F} \Lambda(\mathrm{X}))$ is a fuzzy Alexandroff space.

\subsection{Definition}

The fuzzy Alexandroff space $(\mathrm{X}, \mathrm{F} \Lambda(\mathrm{X}))$ is called the $\Lambda$ - fuzzy Alexandroff space.

\section{Fuzzy $\Lambda_{\gamma}$-Sets and Related Results}

\subsection{Definition}

Let $\mu$ be a fuzzy subset of a fts $(\mathrm{X}, \tau)$. Then the fuzzy $\gamma$-kernel of $\mu$ is denoted by $\Lambda_{\gamma}(\mu)$ and defined as below:

$\Lambda_{\gamma}(\mu)=\wedge\{\eta: \mu \leq \eta, \eta \in \mathrm{F} \gamma \mathrm{O}(\mathrm{X})\}$.

We define $\mathrm{V}_{\gamma}(\mu)=\vee\{\beta: \beta \leq \mu, \mu \in \mathrm{F} \gamma \mathrm{C}(\mathrm{X})\}$.

\subsection{Lemma}

For any fuzzy subsets $\mu, \beta$ and $\left\{\mu_{i}: \mathrm{i} \in \Gamma\right\}$ of a fts $(\mathrm{X}, \tau)$ the following properties hold:

(i) $\Lambda_{\gamma}\left(0_{X}\right)=0_{X}$ and $\Lambda_{\gamma}\left(1_{X}\right)=1_{X}$.

(ii) $\mu \leq \Lambda_{\gamma}(\mu)$.

(iii) $\mu \leq \beta \Rightarrow \Lambda_{\gamma}(\mu) \leq \Lambda_{\gamma}(\beta)$.

(iv) $\Lambda_{\gamma}\left(\Lambda_{\gamma}(\mu)\right)=\Lambda_{\gamma}(\mu)$.

(v) If $\mu \in \mathrm{F} \gamma \mathrm{O}(\mathrm{X})$, then $\mu=\Lambda_{\gamma}(\mu)$.

(vi) $\Lambda_{\gamma}\left(\vee \mu_{i}: \mathrm{i} \in \Gamma\right)=\vee\left\{\Lambda_{\gamma}\left(\mu_{i}\right): \mathrm{i} \in \Gamma\right\}$.

(vii) $\Lambda_{\gamma}\left(\wedge \mu_{i}: \mathrm{i} \in \Gamma\right) \leq \wedge\left\{\Lambda_{\gamma}\left(\mu_{i}\right): \mathrm{i} \in \Gamma\right\}$.

(viii) $\Lambda_{\gamma}\left(1_{X}-\mu\right)=1_{X}-\mathrm{V}_{\gamma}(\mu)$.

Proof: The entire proof is similar to that of Lemma 2.1 in section 2 along with the definition of fuzzy $\Lambda_{\gamma}$-set.

\subsection{Definition}

Let $\mu$ be the fuzzy set of a fts $(\mathrm{X}, \tau)$. Then $\mu$ is called

(i) a fuzzy $\Lambda_{\gamma}$-set if $\mu=\Lambda_{\gamma}(\mu)$,

(ii) a fuzzy $\mathrm{V}_{\gamma}$-set if $\mu=\mathrm{V}_{\gamma}(\mu)$.

The class of all fuzzy $\Lambda_{\gamma}$-sets in a fts $(\mathrm{X}, \tau)$ is denoted by $\mathrm{F} \Lambda_{\gamma}(\mathrm{X})$.

Note: One can easily verify that the concepts of $\Lambda$-fuzzy set and fuzzy $\Lambda_{\gamma^{-}}$ set are independent of each other, since the notion of open set and $\gamma$-open set are completely independent of each other. 


\subsection{Theorem}

For a fts $(\mathrm{X}, \tau)$, the pair $\left(\mathrm{X}, \mathrm{F} \Lambda_{\gamma}(\mathrm{X})\right)$ is a fuzzy Alexandroff space.

Proof: The proof is straightforward from the above lemma 3.2.

\subsection{Remark}

The fuzzy Alexandroff spaces obtained in theorem 2.3 of section 2 and Theorem 3.4 of this section respectively, are independent of each other as so is fuzzy open set and fuzzy $\gamma$-open set.

\subsection{Definition}

The space $\left(\mathrm{X}, \mathrm{F} \Lambda_{\gamma}(\mathrm{X})\right)$ is called the fuzzy $\Lambda_{\gamma}$-Alexandroff space which is termed as fuzzy Independent Alexandroff space.

\subsection{Remark}

Every fuzzy $\Lambda_{\gamma}$-set is a fuzzy $\Lambda_{b}$-set, but the converse is not true as every fuzzy b-open set is not a fuzzy $\gamma$-open set in general and it follows from the example illustrated below:

\subsection{Example}

Let $X=\{x, y\}$ and $\tau=\left\{\{(x, 0.3),(y, 0.6)\},\{(x, 0.2),(y, 0.9)\},\{(x, 0.3),(y, 0.9)\},\{(x, 0.3),(y, 0.6)\}, 0_{X}, 1_{X}\right\}$. We have $\operatorname{FPO}(\mathrm{X})=\{\{(x, p),(y, q)\}$, where $p>0.8$ or $q>0.4\}$. Here, $\{(x, 0.1),(y, 0.5)\}$ is a fuzzy $\Lambda_{b}$-set, but it fails to be a fuzzy $\Lambda_{\gamma}$ -set.

\subsection{Definition}

A fts $(\mathrm{X}, \tau)$ is called $\mathrm{F} \gamma \mathrm{T}_{1}$ space if for any two distinct points $x_{p}, y_{q} \in \mathrm{X}$, there exist fuzzy $\gamma$ neighborhood $\beta$ and $\delta$ in $\mathrm{X}$ such that $\beta\left(y_{q}\right)=0, \beta\left(x_{p}\right)>0$ and $\delta\left(x_{p}\right)=0, \delta\left(y_{q}\right)>0$.

\subsection{Remark}

Every fuzzy singleton set $\left\{x_{p}\right\}$ in $\mathrm{F} \gamma-\mathrm{T}_{1}$ space is a fuzzy $\gamma$-closed. 


\subsection{Theorem}

Let $(\mathrm{X}, \tau)$ be a $\mathrm{F} \gamma-\mathrm{T}_{1}$ space . Then every fuzzy subset of $\mathrm{X}$ is fuzzy $\Lambda_{\gamma}$ -set.

Proof: Let $\mu$ is any fuzzy subset in $\mathrm{X}$ such that $\mu\left(x_{p}\right)=0$.Now, since $\mathrm{X}$ is an $\mathrm{F} \gamma-\mathrm{T}_{1}$ space, then $x_{p}^{c}$ is a fuzzy $\gamma$-open set which follow from the above remark. Therefore, $\Lambda_{\gamma}(\mu) \leq x_{p}^{c} \Rightarrow \Lambda_{\gamma}\left(\mu\left(x_{P}\right)\right)=0$. Thus $\Lambda_{\gamma}(\mu) \leq \mu$.Hence the proof.

\subsection{Remark}

As the fuzzy open set and fuzzy $\gamma$-open set are independent to each other in a fts, therefore we can conclude that a $\mathrm{F} \gamma-\mathrm{T}_{1}$ space is not necessarily a $\mathrm{FT}_{1}$ space and vice versa.

\subsection{Definition}

Let $f: X \rightarrow Y$ be a function from a fts $(\mathrm{X}, \tau)$ into a fts $(\mathrm{Y}, \sigma)$. Then the function $f$ is called fuzzy $\Lambda_{\gamma}$ (resp. $\mathrm{V}_{\gamma}$ )-continuous if $f^{-1}(\beta)$ is a fuzzy $\Lambda_{\gamma}\left(\right.$ resp. $\left.\mathrm{V}_{\gamma}\right)$-set of $\mathrm{X}$ for each $\beta \in \sigma$.

\subsection{Theorem}

Let $f: X \rightarrow Y$ be a function from a fts $(\mathrm{X}, \tau)$ into a fts $(\mathrm{Y}, \sigma)$. Then the following conditions are equivalent:

(i) fis fuzzy $\Lambda_{\gamma}$-continuous function.

(ii) Inverse image of the fuzzy closed set in $\mathrm{Y}$ is a fuzzy $\mathrm{V}_{\gamma}$-set in $\mathrm{X}$.

(iii) $f\left(\mathrm{~V}_{\gamma}(\alpha)\right) \leq \operatorname{cl} f(\alpha)$,for any fuzzy subset $\alpha$ of $\mathrm{X}$.

(iv) $\left(\mathrm{V}_{\gamma}\left(f^{-1}(\delta)\right) \leq f^{-1}(\operatorname{cl}(\delta))\right.$, for any fuzzy subset $\delta$ of $\mathrm{Y}$.

(v) $\left(\Lambda_{\gamma}\left(f^{-1}\right)\left(\delta^{C}\right)\right) \geq f^{-1}\left(\operatorname{int}\left(\delta^{C}\right)\right)$.

Proof: (i) $\Rightarrow$ (ii) Let $\eta$ be any open set in Y. Now, since $f$ is a fuzzy $\Lambda_{\gamma}$ -continuous, thus $f^{-1}(\eta)$ is a $\Lambda_{\gamma}$ - set in X. This implies that $1_{X}-f^{-1}(\eta)$ is a $\left(1_{X}-\Lambda_{\gamma}\right)$ - set in $\mathrm{X}$ that is $f^{-1}\left(\eta^{C}\right)$ is a $\mathrm{V}_{\gamma}$-set in $\mathrm{X}$.

(ii) $\Rightarrow$ (iii) From (ii) we get $\mathrm{V}_{\gamma}\left(f^{-1} \operatorname{cl}(f(\mathrm{~A}))\right)=f^{-1}(\operatorname{cl}(f(\mathrm{~A}))$.

Now $\mathrm{V}_{\gamma}(\mathrm{A}) \leq \mathrm{V}_{\gamma}\left(f^{-1}(f(\mathrm{~A}))\right.$

$\Rightarrow \mathrm{V}_{\gamma}(\mathrm{A}) \leq \mathrm{V}_{\gamma}\left(\mathrm{cl}\left(f^{-1}(f(\mathrm{~A}))\right)\right.$

$=f^{-1}(\operatorname{cl}(f(\mathrm{~A})))$

Thus, $f\left(\mathrm{~V}_{\gamma}(\alpha)\right) \leq \operatorname{cl} f(\alpha)$.

(iii) $\Rightarrow$ (iv) For any fuzzy subset $\delta$ in Y, (iv) can be obtained from (iii). 
(iv) $\Rightarrow$ (v) If we take complement on both sides of (iv) then (v) can be derived easily.

$(v) \Rightarrow$ (i) As the intersection of any two fuzzy $\Lambda_{\gamma}$-set is fuzzy $\Lambda_{\gamma}$-set, thus by (v) we can say that $\mathrm{f}$ is a fuzzy $\Lambda_{\gamma}$-continuous.

\subsection{Remark}

Every fuzzy $\Lambda_{\gamma}$-continuous function is a fuzzy $\Lambda_{b^{-}}$continuous function. But the converse is not true and that follows from the above remark 3.7.

\section{Generalization of Fuzzy $\Lambda$ - Closed Sets}

\subsection{Definition}

A fuzzy subset $\lambda$ of a fts $(\mathrm{X}, \tau)$ is called fuzzy $\Lambda$-closed set if $\lambda=\mu \wedge \delta$, where $\mu$ is a $\Lambda$-fuzzy set and $\delta$ is a fuzzy closed set. The family of all fuzzy $\Lambda$ -closed sets is denoted by $\mathrm{F} \Lambda \mathrm{C}(\mathrm{X})$.

A fuzzy subset $\lambda$ of a fts $(\mathrm{X}, \tau)$ is called fuzzy $\Lambda$-open if its complement is fuzzy $\Lambda$-closed set.

\subsection{Remark}

Every fuzzy locally closed set is a fuzzy $\Lambda$-closed set .

\subsection{Theorem}

Every fuzzy generalized closed set of a fts $(\mathrm{X}, \tau)$ is a fuzzy closed set iff it is fuzzy $\Lambda$-closed set.

Proof: Let $\lambda$ be a fuzzy generalized closed set. Thus $\operatorname{cl}(\lambda \leq \mu)$, whenever $\lambda \leq \mu$, where $\mu \in \tau$.

Now, since $\lambda$ is fuzzy $\Lambda$-closed set, then $\lambda=\Lambda(\lambda) \wedge \operatorname{cl}(\lambda)$, since $\Lambda(\lambda) \geq \operatorname{cl}(\lambda)$. Therefore, $\lambda$ is a fuzzy closed set.

Converse part follows directly from the fact that every fuzzy closed set is a fuzzy $\Lambda$-closed set.

\subsection{Proposition}

In a fts $(\mathrm{X}, \tau)$, the following conditions are equivalent:

(i) $(\mathrm{X}, \tau)$ is fuzzy $\mathrm{T}_{1 / 2}$ space.

(ii) Every fuzzy generalized closed set is a fuzzy $\Lambda$ - closed set. 
Proof: (i) $\Rightarrow$ (ii) Given $(\mathrm{X}, \tau)$ be a fuzzy $\mathrm{T}_{1 / 2}$ space.Hence, from the definition we have that every fuzzy generalized closed set is fuzzy closed set and it is a fuzzy $\Lambda$-closed set.

Proof: (ii) $\Rightarrow$ (i) Using theorem 3.14, it can be proved easily.

\subsection{Definition}

A fuzzy subset $\lambda$ of a fts $(\mathrm{X}, \tau)$ is called fuzzy $\Lambda_{\gamma}$-closed set if $\lambda=\mu \wedge \delta$ ,where $\mu$ is a fuzzy $\Lambda_{\gamma}$-set and $\delta$ is an fuzzy closed set. The family of all fuzzy $\Lambda_{\gamma}$-closed sets is denoted by $\mathrm{F} \Lambda_{\gamma} \mathrm{C}(\mathrm{X})$.

The fuzzy subset $\lambda$ of the fts $(\mathrm{X}, \tau)$ is called a fuzzy $\Lambda_{\gamma}$-open if its complement is fuzzy $\Lambda_{\gamma^{-}}$closed set.

\subsection{Lemma}

For a fuzzy subset $\lambda$ of a fts ( $\mathrm{X}, \tau$ ), the following conditions are equivalent :

(i) $\lambda$ is a fuzzy $\Lambda_{\gamma}$-closed set.

(ii) $\lambda=\mu \wedge \operatorname{cl}(\lambda)$, where $\mu$ is a fuzzy $\Lambda_{\gamma}$-set .

(iii) $\lambda=\Lambda_{\gamma}(\lambda) \wedge \operatorname{cl}(\lambda)$.

Proof: (i) $\Rightarrow$ (ii) Let $\lambda$ be a fuzzy $\Lambda_{\gamma}$-closed set.

Therefore, $\lambda=\mu \wedge \delta$, where $\mu$ is a fuzzy $\Lambda_{\gamma}$-set and $\delta$ is an fuzzy closed set. Since $\lambda \leq \delta$ implies $\operatorname{cl}(\lambda) \leq \delta$ and

$\lambda=\mu \wedge \delta \geq \mu \wedge \operatorname{cl}(\lambda) \geq \lambda$.

Therefore, we have $\lambda=\mu \wedge \operatorname{cl}(\lambda)$.

(ii) $\Rightarrow$ (iii) Let $\lambda=\mu \wedge \operatorname{cl}(\lambda)$, where $\mu$ is a fuzzy $\Lambda_{\gamma}$-set.

Since $\lambda \leq \mu$ and it implies that

$\Lambda_{\gamma}(\lambda) \leq \mu$ and $\lambda=\mu \wedge \delta \geq \Lambda_{\gamma}(\lambda) \wedge \operatorname{cl}(\lambda) \geq \lambda$.

Therefore, we have $\lambda=\Lambda_{\gamma}(\lambda) \wedge \operatorname{cl}(\lambda)$.

(iii) $\Rightarrow$ (i) It is obvious and hence omitted.

\subsection{Remark}

Fuzzy $\Lambda_{\gamma}$-closed set and fuzzy $\Lambda$-closed set are independent of each other , since every fuzzy $\Lambda_{\gamma}$-set and $\Lambda$-fuzzy set are independent. 


\subsection{Proposition}

If $\lambda$ be any fuzzy dense set and fuzzy $\Lambda_{\gamma}$-closed set, then $\lambda$ is fuzzy $\Lambda_{\gamma}$-set.

Proof: Let $\Lambda$ be any fuzzy dense set and fuzzy $\Lambda_{\gamma}$ closed set, then $\Lambda=\Lambda_{\gamma}(\lambda) \wedge \operatorname{cl}(\lambda)=\Lambda_{\gamma}(\lambda) \wedge 1_{X}=\Lambda_{\gamma}(\lambda)$. Thus, $\lambda$ is a fuzzy $\Lambda_{\gamma}$-set.

\subsection{Definition}

A fuzzy subset $\lambda$ in a fts $(\mathrm{X}, \tau)$ is said to be fuzzy semi $\gamma$-closed set if $\operatorname{int}_{\gamma}(\operatorname{cl}(\lambda)) \leq \lambda$.

\subsection{Proposition}

A fuzzy $\Lambda_{\gamma}$-closed set is fuzzy closed set if it is fuzzy semi $\gamma$ - closed set.

Proof: Let $\lambda$ be any fuzzy $\Lambda_{\gamma}$-closed set, so $\lambda=\Lambda_{\gamma}(\lambda) \wedge \operatorname{cl}(\lambda)$. Given that $\lambda$ is a fuzzy semi $\gamma$ - closed set. Thus $\operatorname{int}_{\gamma}(\operatorname{cl}(\lambda)) \leq \lambda$.

Let $\operatorname{int}_{\gamma}(\operatorname{cl}(\lambda)) \leq \lambda \leq \delta_{k}, \mathrm{k} \in \mathrm{I}$ where $\delta_{k}$ are fuzzy $\gamma$-open set. Therefore, $\operatorname{cl}(\lambda) \leq \wedge \delta_{k}$.

This implies that $\mathrm{cl}(\lambda) \leq \Lambda_{\gamma}(\lambda)$.

Therefore, $\lambda=\operatorname{cl}(\lambda)$ and hence $\lambda$ is a fuzzy closed set.

\subsection{Definition}

The fuzzy set $\lambda$ of a fts $(\mathrm{X}, \tau)$ is called a fuzzy $\Lambda_{\gamma}$-generalized closed set if $\mathrm{cl}(\lambda) \leq \mu$ whenever $\left(\lambda \leq \mu\right.$ and $\mu$ is fuzzy $\Lambda_{\gamma}$-open set.

\subsection{Proposition}

In a fts $(\mathrm{X}, \tau)$ the union of any two fuzzy $\Lambda_{\gamma}$-generalized closed sets is again a fuzzym $\Lambda_{\gamma}$-generalized closed set.

Proof: Let $\alpha$ and $\beta$ be any two fuzzy $\Lambda_{\gamma}$-generalized closed sets in X. Thus $\operatorname{cl}(\alpha) \leq \mu$,whenever $\alpha \leq \mu$ and $\operatorname{cl}(\beta) \leq \mu$, whenever $\beta \leq \mu$, where $\mu$ is fuzzy $\Lambda_{\gamma}$-open set .

Now, if $\alpha \vee \beta \leq \mu$, then $\operatorname{cl}(\alpha \vee \beta)=\operatorname{cl}(\alpha) \vee \operatorname{cl}(\beta) \leq \mu$. Hence the proof is done.

\subsection{3. remark}

In a fts $(\mathrm{X}, \tau)$, the intersection of any two fuzzy $\Lambda_{\gamma}$-generalized closed sets may not be a fuzzy $\Lambda_{\gamma}$ generalized closed set as seen in the following example. 


\subsection{4. example}

Let $X=\{x, y\}$ and

$\tau=\left\{\{(x, 0.8),(y, 0.3)\}, 0_{X}, 1_{X}\right\}$.

We have $\mathrm{F} \gamma \mathrm{O}(\mathrm{X})=\{\{(x, p),(y, q)\}$, where $q>0.3$ for any value of $p\}$.

Here, $\{(x, 0.6),(y, 0.2)\}$ and $\{(x, 0.3),(y, 0.25)\}$ are two fuzzy $\Lambda_{\gamma}$-generalized closed sets, but their intersection is not a fuzzy $\Lambda_{\gamma}$-generalized closed set.

\subsection{Definition}

Let $f: X \rightarrow Y$ be a function from a fts $(\mathrm{X}, \tau)$ into a fts $(\mathrm{y}, \sigma)$. Then the function $f$ is called a fuzzy $\Lambda_{\gamma}$-generalized continuous, if $f^{-1}(\mu)$ is fuzzy $\Lambda_{\gamma}$-generalized closed in $\mathrm{X}$ for each fuzzy closed $\mu$ in $\mathrm{Y}$.

\subsection{Proposition}

Every fuzzy closed set is a fuzzy $\Lambda_{\gamma}$-generalized closed set in a fts $(\mathrm{X}, \tau)$.

Proof: Let $\beta$ be any fuzzy closed set and $\mu$ be any fuzzy $\Lambda_{\gamma}$-open set such that $\beta \leq \mu, \operatorname{cl}(\beta)=\beta \leq \mu$.Thus $\beta$ is a fuzzy $\Lambda_{\gamma}$-generalized closed set.

\subsection{Remark}

It is clear from the above example 4.14 that in a fts $(\mathrm{X}, \tau)$, every fuzzy $\Lambda_{\gamma}$-generalized closed set is not a fuzzy closed set .

\subsection{Remark}

Every fuzzy $\Lambda_{\gamma}$-closed set need not be a fuzzy $\Lambda_{\gamma}$-generalized closed set as verified in the following example.

\subsection{9. example}

Let $X=\{x, y\}$ and

$\tau=\left\{\{(x, 0.2),(y, 0.2)\}, 0_{X}, 1_{X}\right\}$.

Here $\mathrm{F} \gamma \mathrm{O}(\mathrm{X})=\{\{(x, p),(y, q)\}$, where $0 \leq p \leq 0.2$ and $p>0.8\}$.

Let us suppose that

$\lambda=\{(x, 0.85),(y, 0.85)\}$ and we have $\Lambda_{\gamma}(\lambda)=\lambda$ which implies that $\lambda$ is a fuzzy $\Lambda_{\gamma}$-closed set. Again, $\lambda \leq \lambda$ and $\operatorname{cl}(\lambda)=1_{X} \lambda$. Therefore, $\lambda$ is not fuzzy $\Lambda_{\gamma^{-}}$generalized closed set. 


\subsection{0. remark}

Using the above example, we see that the fuzzy $\operatorname{set}\{(x, 0.3),(y, 0.25)\}$ is a fuzzy $\Lambda_{\gamma}$-generalized closed set but not a fuzzy $\Lambda_{\gamma}$-closed set .

\section{Applications}

i) B. Bhattacharya has shown that the collection of all $\gamma$-open sets forms an independent topology in a fts. But in the present paper, we show that the collection of all $\Lambda_{\gamma}$-sets forms a stronger independent topology, called fuzzy $\Lambda_{\gamma}$-Alexandroff space. Thus, this structure may be used for further work on fuzzy independent topology and some related areas.

ii) Also the present study may have application in computational fuzzy topology or in quantum Physics, particularly in connection with string theory and $\epsilon^{\infty}$ theory, etc. and many more as fuzzy topology may be relevant to those concepts .

iii) Generalized closed sets play an important role in the study of morphology. We contemplate that the generalized forms of fuzzy $\gamma$-closed sets may be relevant in the study of grey-scale connected operator and infstructuring function. 
From the above discussion, we can draw the following figures in a fuzzy topological space
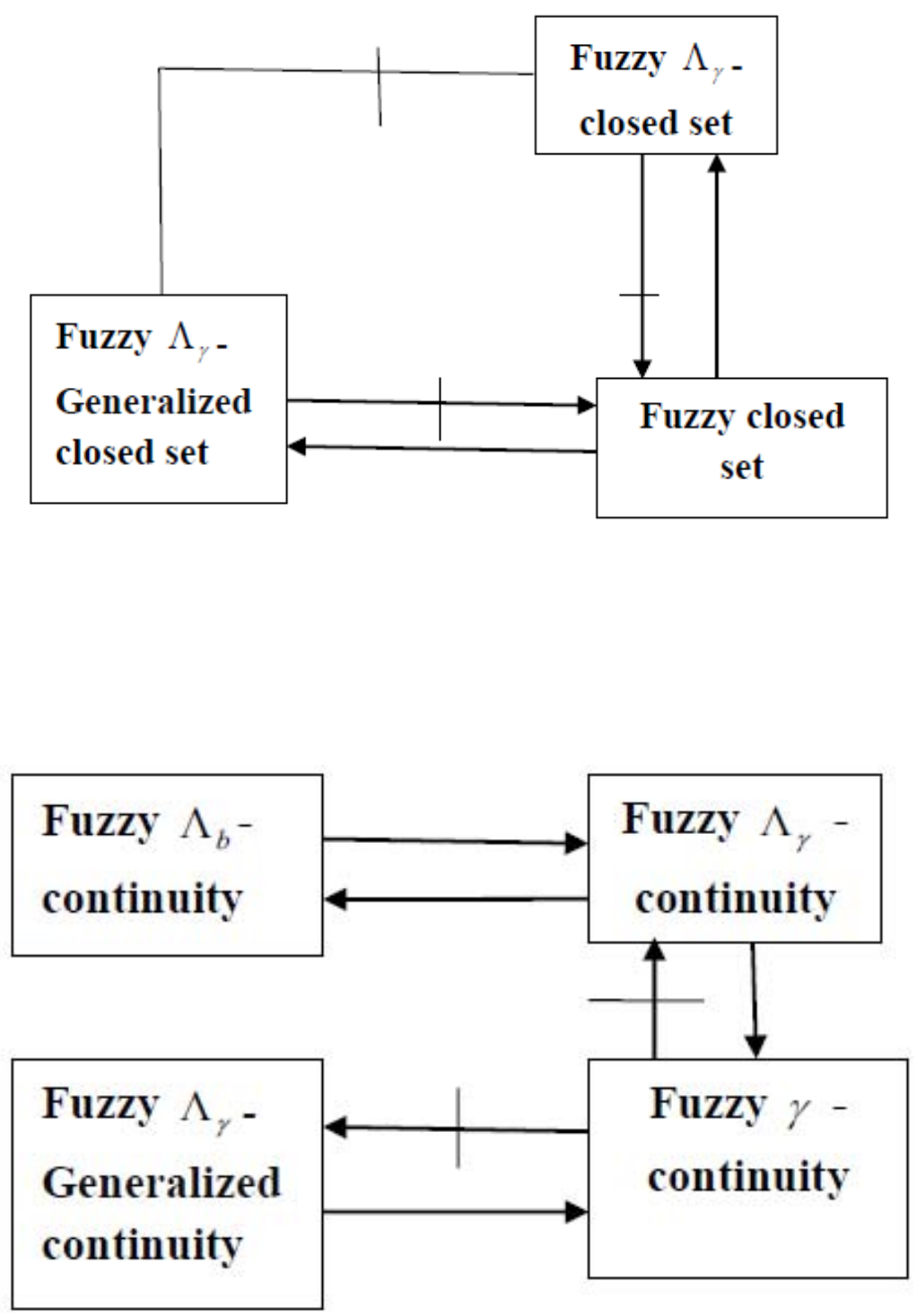


\section{Conclusion}

Undoubtedly the worth of fuzzy topology has rapidly been appeared in many fields of both pure and applied sciences where fuzzy open set and fuzzy continuity are playing the key role in the structure of fuzzy topology and as a consequence, the weaker form of fuzzy continuity and open sets have been studied by many researchers. This paper presents a parallel form of fuzzy topology and it is a mathematical existence of parallel topology rather for parallel circuits. Frontier may be done as an application of fuzzy contra $\gamma$-continuity in the near future.

\section{References}

[1] D. Andrijevic and M. Ganster, On PO-equivalent topologies, in IV International Meeting on Topology in Italy (Sorrento, 1988), Rend. Circ. Mat. Palermo (2) Suppl., 24, pp. 251-256, (1990).

[2] G. Aslim and G. Gunel , On fuzzy $\Lambda_{b}$-sets and fuzzy $\Lambda_{b}$-continuity, Chaos, Solitons and Fractals, 42, pp. 1024-1030, (2009).

[3] K. K. Azad, Fuzzy semi continuity, fuzzy almost continuity and weakly continuitys. Math. Anal. Appl., 82, pp. 14-32, (1981).

[4] G. Balasubramanian and P. Sundaram, On some generalizations of fuzzy continuous functions, Fuzzy Sets and Systems, 86, pp. 93-100, (1997).

[5] B. Bhattacharya and J. Chakraborty, Generalized regular fuzzy locally closed sets and some generalizations of fuzzy LC-continuous functions, Mathematical Sciences International Research Journal 3 (1), pp. 397399, (2014).

[6] B.Bhattacharya,Fuzzy independent topological spaces generated by fuzzy $\gamma$-open sets and their application, accepted in Afrika Matematika, (2017).

[7] C. L. Chang, Fuzzy topological spaces, J. Math. Anal. Appl., 24, pp. 338-353, (1968). 
[8] A. Csaszar, Generalized topology, generalized continuity. Acta Math. Hungar, 96, pp. 351-357,(2002).

[9] A. Csaszar, Generalized open sets in generalized topologies, Acta Math. Hungar, 106, pp. 53-66, (2005).

[10] A. Csaszar, $\delta$-and $\theta$-modification of generalized topologies, Acta Math. Hungar, 120, pp. 275-279, (2008).

[11] E. Ekici, On (LC, S)-continuous functions, Chaos, Solitons and Fractals, 38, pp. 430-438, (2001).

[12] S. M. Jafarian Amiri, A. Jafarzadeh and H. Khatibzade, An Alexandroff Topology on graphs, Bull. Of the Iranian Math. Soc., 39, pp. 647-662, (2013).

[13] A. Keskin and T. Noiri, Almost contra g-continuous function, Chaos, Solitons and Fractals, 42, pp. 238-246, (2013).

[14] M. S. El Naschie, On the certification of heterotic strings, theory and $\epsilon^{\infty}$ theory, Chaos, Solitons and Fractals, 2, pp. 397- 408, (2000).

[15] M. S. El Naschie, On a fuzzy Kahler-likl manifold which is consistent with the two slit experiment, Int. J. Nonlinear SciNumerSimul 205.

[16] G. Palani Chetty, Generalized Fuzzy Topology, Italian J. Pure Appl. Math., 24, pp. 91-96, (2008).

[17] A. Paul, B. Bhattacharya and J. Chakraborty, On $\Lambda^{\gamma}$ - Set in Fuzzy Bitopological Spaces, Bol. Soc. Paran Mat. 35, n.3, pp. 285-299, (2017).

[18] A. Paul, B. Bhattacharya and J. Chakraborty, On $\gamma$-hyperconnectedness and fuzzy mappings in fuzzy bitopological spaces, Journal of Intelligent and Fuzzy Systems, 32, n. 3, pp. 1815-1820, (2017).

[19] M. K. Prakash Singal, Fuzzy pre-open sets and fuzzy pre separation axiom. Fuzzy Sets and system, 44, pp. 273-281, (1991).

[20] M. E. El Shafei and A. Zakari, $\theta$-Generalized closed sets in fuzzy topological spaces, The Arabian Journal for Science and Engineering 31, N 24, (2006).

[21] S. S. Thakur and S. Singh, On fuzzy semi-pre-open sets and fuzzy semipro-continuity, Fuzzy Sets and system 98, pp. 383-391, (1998). 
[22] G. Thangaraj and G. Balasubramanian, On Somewhat fuzzy continuous functions, J. Fuzzy Math. 11 (2), pp. 725- 736, (2003).

[23] C. K. Wong, Fuzzy points and local properties of fuzzy topology, Journal of Mathematical Analysis and Applications, 46, pp. 316-328, (1974).

[24] L. A. Zadeh, Fuzzy sets. Inform. And Control, 8, 338-353, (1965).

[25] B. S. Zhong, Fuzzy strongly semi-open set and fuzzy strongly semicontinuity, Fuzzy Sets and system, 52, pp. 345-351, (1992).

\title{
Baby Bhattacharya
}

Department of Mathematics, National Institute of Technology,

Agartala, 799046,

India

e-mail : babybhatt75@gmail.com

\section{Arnab Paul}

Science and Humanities Department, Techno College of Engineering Agartala, Agartala, 799004, India

e-mail : mrarnabpaul87@gmail.com

and

\author{
Jayasree Chakraborty \\ Department of Mathematics, \\ National Institute of Technology, \\ Agartala, 799046, \\ India \\ e-mail : chakrabortyjaysree1@gmail.com
}

